\title{
Dynamical mutation of dark energy
}

\author{
L. R. Abramo, ${ }^{1}$ R. C. Batista, ${ }^{1}$ L. Liberato, ${ }^{2}$ and R. Rosenfeld ${ }^{2}$ \\ ${ }^{1}$ Instituto de Física, Universidade de São Paulo, CP 66318, 05315-970, São Paulo, Brazil \\ ${ }^{2}$ Instituto de Física Teórica, Universidade Estadual Paulista, R. Pamplona 145, 01405-900, São Paulo, Brazil
}

(Received 19 October 2007; published 6 March 2008)

\begin{abstract}
We discuss the intriguing possibility that dark energy may change its equation of state in situations where large dark energy fluctuations are present. We show indications of this dynamical mutation in some generic models of dark energy.
\end{abstract}

DOI: 10.1103/PhysRevD.77.067301

PACS numbers: 95.36.+x, 98.80.Cq

\section{INTRODUCTION}

There is ample evidence that supports the existence of dark matter (DM) and dark energy (DE) in the Universe [1]. Because of its charge neutrality and dustlike equation of state (i.e., negligible pressure), dark matter starts to cluster gravitationally very early in the history of the Universe and is crucial for the formation of large scale structures. Dark energy, on the other hand, becomes relevant only more recently and is presumed to be a smooth component with a negative equation of state in order to fuel the accelerated expansion of the Universe [2].

At the background level, dark energy (or any other fluid) is completely determined by its equation of state $w=$ $p_{e} / \rho_{e}$, where $p_{e}$ is the pressure and $\rho_{e}$ is the energy density of dark energy. Already at this level, dark energy can affect large scale structures [3] — see also [4] for the effect in different parametrizations of the equation of state of dark energy.

However, if dark energy is in fact a manifestation of a dynamical mechanism such as a scalar field, then it will also develop inhomogeneities due to its gravitational interactions with itself and with dark matter [5]. In linear perturbation theory, besides the energy density perturbation $\delta \rho$, we need two extra degrees of freedom to characterize cosmological perturbations: the pressure perturbation $\delta p$ and the scalar anisotropic stress $\pi[6,7]$. Alternatively, one can also use the velocity potential $\theta=$ $\vec{\nabla} \cdot \vec{v}$ and the anisotropic stress [8].

The inhomogeneities of dark energy are often quite small, particularly in the case of ordinary (canonical) scalar field models with almost $\Lambda$-like behavior-that is, when $w \simeq-1[5,9,10]$. In fact, as $w \rightarrow-1$ the perturbations in all dark energy models are suppressed in relation to those of dark matter. However, if that is not the case then the dark energy density contrast $\delta_{e} \equiv \delta \rho_{e} / \rho_{e}$ can be either small or large, depending on the pressure perturbations of dark energy [11].

Here we present further evidence of an intriguing possibility which was first pointed out in supergravitymotivated scalar field models of dark energy $[12,13]$ : that dark energy can mutate into a fluid with clustering properties similar to those of dark matter. We will show that this effect is a generic feature of dark energy and that it has a simple origin: when pressure perturbations are large, the effective equation of state inside a collapsed region can be completely different from the equation of state of its homogeneous component.

\section{GRAVITATIONAL COLLAPSE WITH DARK ENERGY}

In the following, in order to specify the properties of the dark energy perturbations we will neglect the anisotropic stress. Moreover, we will characterize the pressure perturbation in a simplified manner, using the so-called "effective (or nonadiabatic) sound velocity" [14], defined as $c_{\text {eff }}^{2} \equiv \delta p_{e} / \delta \rho_{e}$, which we will assume to be a function of time only. Notice that this assumption lacks a formal basis in perturbation theory, since $\delta p_{e}$ is a perturbed variable whose time and spatial dependences can be, and often are, independent of the variations of $\delta \rho_{e}$. Nevertheless, in a particular gauge (the so-called "rest frame" of the fluid, where $T_{0}^{i}=0$ ), the effective sound speed coincides with the phase velocity of linear relativistic perturbations $c_{X}^{2}[14,15]$.

Describing the pressure perturbation as $\delta p_{e}=c_{\text {eff }}^{2} \delta \rho_{e}$ allows us to treat a wide variety of dark energy models and, crucially, it also allows us to compute nonlinear structure formation using the spherical collapse (SC) model [16]. Furthermore, in this case the SC equations (derived in a simplified relativistic framework) are identical to the equations of pseudo-Newtonian cosmology [17]. This means that the physics of gravitational collapse of structures such as galaxy clusters is well described within this framework.

Consider then, in the spirit of the SC model, a spherically symmetric region of constant dark energy overdensity (the so-called "top-hat" density profile.) Let us call $\rho_{e}^{c}=$ $\rho_{e}+\delta \rho_{e}$ and $p_{e}^{c}=p_{e}+\delta p_{e}$ the energy density and the pressure of this region, which are modified with respect to the corresponding background quantities $\rho_{e}$ and $p_{e}$ by the perturbations $\delta \rho_{e}$ and $\delta p_{e}$. The equation of state $w$ is defined as the ratio of the total pressure to the total energy density and hence it will be different for the background and the interior of the collapsed region. A simple calculation shows that the equation of state inside the collapsed region $w^{c}$ is given by 


$$
w^{c}=\frac{p_{e}+\delta p_{e}}{\rho_{e}+\delta \rho_{e}}=w+\left(c_{\mathrm{eff}}^{2}-w\right) \frac{\delta_{e}}{1+\delta_{e}} .
$$

For small density contrasts $\left|\delta_{e}\right| \ll 1$, the equation of state inside the overdense region does not change appreciably. However, if $c_{\text {eff }}^{2} \neq w$, then in the nonlinear regime, where $\delta \gtrsim 1$ (halos), there could be a substantial modification in $w^{c}$ with respect to the background equation of state. Even in underdense regions (voids), where $\delta \approx-1$, there could be large modifications of the equation of state. Hence, in principle dark energy could even effectively mutate into dark matter inside halos and voids.

The above argument is completely general. What remains to be shown is whether there are models in which this dramatic situation is actually realized. This requires a nonlinear analysis of the evolution of perturbations for two gravitationally coupled fluids, dark energy and dark matter (we will neglect radiation and baryons in what follows). Unfortunately, at present there are no totally rigorous methods for performing this analysis - except in the case of canonical scalar fields, but even then only approximately $[9,10]$.

Here we employ a generalization of the SC model for the case of a relativistic fluid with pressure. In the next section we present the relevant equations and mention under which conditions they are equivalent to a pseudo-Newtonian approach. We will then analyze the evolution of the coupled system of perturbations for a wide variety of dark energy models for which the pressure perturbations are characterized by some homogeneous effective sound speed.

\section{NONLINEAR EVOLUTION}

We define $H=\dot{a} / a$ and $h=\dot{r} / r$ as the expansion rates for the background ( $a$ is the scale factor) and for the perturbed region ( $r$ is the size of the collapsing region), respectively. In what follows we work within two assumptions, namely: that there is no nongravitational interaction between DE and DM, and that the total energy of both DE and DM contained in the collapsed region is constant. The possibility of including DM-DE interactions in the study of structure formation was studied in $[13,18]$ and a discussion of possible outflow of energy from the collapsed region can be found in $[12,19]$.

Using the continuity equations for the background and for the perturbed region for a fluid species $j$,

$$
\frac{\dot{\rho}_{j}}{\rho_{j}}=-3 H\left(1+w_{j}\right), \quad \frac{\dot{\rho}_{j}^{c}}{\rho_{j}^{c}}=-3 h\left(1+w_{j}^{c}\right),
$$

and we obtain, for the density contrasts:

$$
\dot{\delta}_{j}=-3\left(1+\delta_{j}\right)\left[h\left(1+w_{j}^{c}\right)-H\left(1+w_{j}\right)\right] .
$$

Obviously, for matter we have $w_{m}=w_{m}^{c}=0$.

Using Eq. (1) and the fact that the local expansion rate is related to the velocity field in the perturbed region by $h=$ $H+\theta / 3 a$, we arrive at

$$
\dot{\delta}_{j}+3 H\left(c_{j \mathrm{eff}}^{2}-w_{j}\right) \delta_{j}+\frac{\theta}{a}\left[\left(1+w_{j}\right)+\left(1+c_{j \mathrm{eff}}^{2}\right) \delta_{j}\right]=0 .
$$

The equation that determines the evolution of $\theta$ comes from the "acceleration" in the perturbed region:

$\dot{\theta}+H \theta+\frac{\theta^{2}}{3 a}+\frac{3}{2} H^{2} a\left[\Omega_{m} \delta_{m}+\left(1+3 c_{\mathrm{eff}}^{2}\right) \Omega_{e} \delta_{e}\right]=0$.

Notice that there is only one equation for the peculiar velocity, even in the case of 2 fluids. This is clearly necessary, because in the $\mathrm{SC}$ model it is a single spherically symmetric region that detaches from the background, with a peculiar expansion rate given by $h=H+\theta / 3 a$. When there is pressure and pressure gradients, relativistic corrections almost surely break this identity between the velocities of the fluids, which means that the SC model with a top-hat profile is inconsistent with a fully relativistic calculation. The assumption that $c_{\mathrm{eff}}^{2}$ is only time dependent implies that $\delta p$ is also only time dependent-which ultimately guarantees the validity of the top-hat SC model.

The SC equations capture many features of the gravitational physics one expects to find at the scale of the collapsed structures we see today. This is because the exact same equations can be derived from a pseudo-Newtonian treatment of perturbations when gradients of pressure can be neglected - as is the present case, of a top-hat profile. When linearized, the ensuing equations correspond to the linear equations from general relativity in the case of $c_{\text {eff }}^{2}=0$ for subhorizon scales [20]. Furthermore, even when $c_{\mathrm{eff}}^{2} \neq 0$, although the equations are not equivalent anymore, still the growing modes of the linearized pseudoNewtonian perturbations are identical to the growing modes of the linearized relativistic perturbations [17].

\section{NUMERICAL SOLUTIONS}

We solved the coupled differential equations for $\delta_{e}, \delta_{m}$, and $\theta$, for a few representative models of dark energy. In our approximation, a dark energy model is determined by its background equation of state and the effective speed of sound. In a previous paper we investigated the particular case where the sound speed of dark energy is equal to its equation of state, $c_{\mathrm{eff}}^{2}=w$, and therefore $w^{c}=w$. In that case, one can see clearly from Eq. (1) that there is no mutation [11].

In this paper we expand our previous analysis to the following cases: $c_{\text {eff }}^{2}=1, c_{\text {eff }}^{2}=0, c_{\text {eff }}^{2}=-1$, and $c_{\text {eff }}^{2}=$ $-w$. The first case is motivated by the common situation when dark energy is modeled by a canonical scalar field, since in the gauge corresponding to the rest frame of the scalar field the effective sound speed is $c_{\mathrm{eff}}^{2}=1$ [14]. The second case (null pressure perturbations) can occur in socalled "silent quartessence" models [21]. The third case represents a perturbation with behavior close to a cosmological constant and the last case reproduces the so-called 


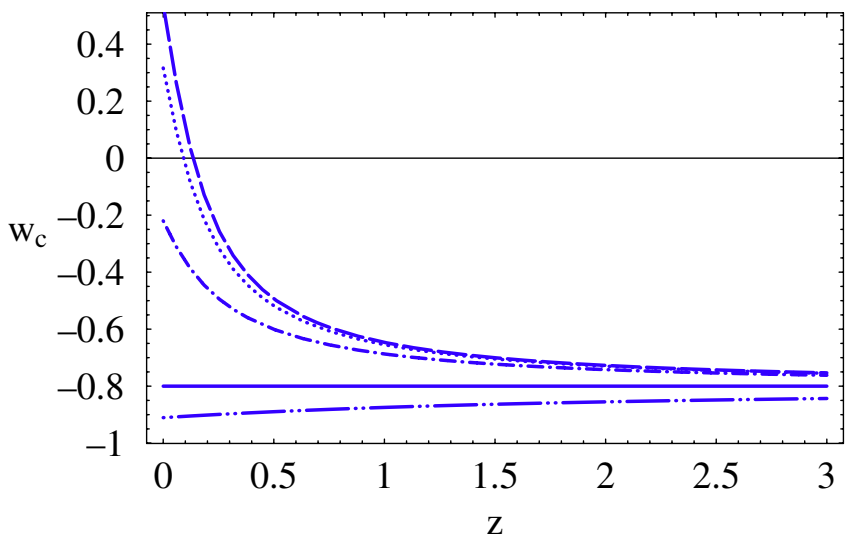

FIG. 1 (color online). Clustered dark energy equation of state for different cases of effective speed of sound for background $w=-0.8: c_{\mathrm{eff}}^{2}=w$ (solid line), $c_{\mathrm{eff}}^{2}=1$ (dashed line), $c_{\mathrm{eff}}^{2}=0$ (dot-dashed line), $c_{\mathrm{eff}}^{2}=-w$ (dotted line), and $c_{\mathrm{eff}}^{2}=-1$ (double dot-dashed line). The instant of turnaround $(h=0)$ is $z=0.3$ for $c_{\mathrm{eff}}^{2}=w, z=0.5$ for both $c_{\mathrm{eff}}^{2}=-w$ and $c_{\mathrm{eff}}^{2}=1$, and $z=0.6$ for $c_{\mathrm{eff}}^{2}=0$ (for $c_{\mathrm{eff}}^{2}=-1$ there is no turnaround).

"generalized Chaplygin gas" in a certain limit $(\alpha=1)$ [22].

We use adiabatic initial conditions for the perturbations $\left[\delta_{e}^{i}=(1+w) \delta_{m}^{i}\right]$ at a redshift $z=1000$, an initial velocity field coincident with the Hubble flow $\left(\theta^{i}=0\right), H_{0}=$ $72 \mathrm{~km} \mathrm{~s}^{-1} \mathrm{Mpc}^{-1}, \Omega_{m}=0.25$, and $\Omega_{e}=0.75$. In the examples shown below, we fixed the background equation of state of dark energy at $w=-0.8$.

In Fig. 1 we show the values of the clustered equation of state $w_{c}$ as a function of the redshift. The initial condition $\delta_{m}^{i}$ was chosen such that the dark energy perturbation $\delta_{e} \sim$ $\mathcal{O}(1)$ today $(z=0)$. The perturbation in dark matter is typically 1 to 2 orders of magnitude larger, which is consistent with the typical density contrast in galaxy clusters, for which $\delta_{m} \sim \mathcal{O}\left(10^{3}\right)$.

As discussed above, for the case $c_{\text {eff }}^{2}=w$ we obtain no mutation in the equation of state in the perturbed region. However, substantial modifications are found for other possibilities of $c_{\mathrm{eff}}^{2}$. The largest modifications arise for $c_{\text {eff }}^{2}=1$, which in our example is very similar to $c_{\text {eff }}^{2}=$ $-w$. In these two cases, even a complete metamorphosis of dark energy into a fluid which clusters as strongly as dark matter is possible at recent epochs, due to the large perturbations inside the collapsed region. The behavior for $c_{\mathrm{eff}}^{2}=$ -1 is also easily understood from Eq. (1), since this is the only case where $c_{\text {eff }}^{2}-w<1$.

As expected, the effect of mutation is greatly reduced for a background equation of state close to that of a cosmological constant, independent of $c_{\mathrm{eff}}^{2}$. We illustrate that fact in Fig. 2, where we show the equation of state inside the collapsing region for different models of clustered dark

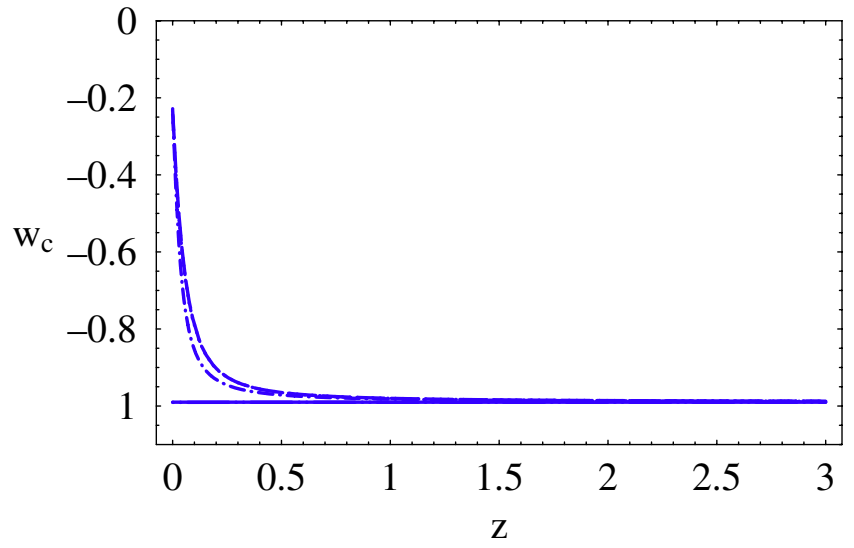

FIG. 2 (color online). Clustered dark energy equation of state for different cases of effective speed of sound for background $w=-0.99$. Lines are the same as in Fig. 1. The cases of $c_{\mathrm{eff}}^{2}=$ $w$ and $c_{\mathrm{eff}}^{2}=-1$, as well as $c_{\mathrm{eff}}^{2}=1$ and $c_{\mathrm{eff}}^{2}=-w$, lie on top of each other. Turnaround occurs at approximately $z \simeq 0.6$ in all cases.

energy, in the case of a background equation of state $w=$ -0.99 , and with the same initial conditions that were used in Fig. 1. Nevertheless, even in this case large density contrasts can still arise in the dark energy component, which lead to the mutation of dark energy.

\section{CONCLUSIONS}

We have shown that it is possible to change radically the clustering properties of dark energy in collapsed regions (halos and voids.) We exemplified this behavior with a few models for the dark energy perturbations and showed that it happens not only in scalar field models but also in generic models of dark energy - in particular the generalized Chaplygin gas and silent quartessence models.

Since the physics of most observed collapsed structures, such as galaxy clusters, is well approximated by quasiNewtonian physics, this dynamical mutation should be a general phenomenon. Clearly, this is a crucial issue for all attempts to compute the influence of dark energy on the formation of large scale structures. More detailed studies, including a relativistic approach and using different realistic parametrizations of the dark energy equation of state, are currently under way [17].

\section{ACKNOWLEDGMENTS}

We would like to thank Ioav Waga for many fruitful discussions. This work has been supported by FAPESP Grants No. 04/13668-0 (L. R. A. and R. R.) and No. 05/ 00554-0 (R. C. B.), a CNPq Grant No. 309158/2006-0 (R. R.) and a CAPES grant (L. L.). 
[1] See, e.g., U. Seljak, A. Slozar, and P. McDonald, J. Cosmol. Astropart. Phys. 10 (2006) 014.

[2] For reviews, see e.g., T. Padmanabhan, Phys. Rep. 380, 235 (2003); P. J. E. Peebles and B. Ratra, Rev. Mod. Phys. 75, 559 (2003); V. Sahni and A. A. Statobinsky, Int. J. Mod. Phys. D 15, 2105 (2006).

[3] See, e.g., L. M. Wang and P. J. Steinhardt, Astrophys. J. 508, 483 (1998); R. A. Battye and J. Weller, Phys. Rev. D 68, 083506 (2003); E. V. Linder, Phys. Rev. D 72, 043529 (2005).

[4] L. Liberato and R. Rosenfeld, J. Cosmol. Astropart. Phys. 07 (2006) 009.

[5] K. Coble, S. Dodelson, and J. A. Frieman, Phys. Rev. D 55, 1851 (1997).

[6] J. M. Bardeen, Phys. Rev. D 22, 1882 (1980).

[7] H. Kodama and M. Sasaki, Prog. Theor. Phys. Suppl. 78, 1 (1984).

[8] C.-P. Ma and E. Bertschinger, Astrophys. J. 455, 7 (1995).

[9] S. Dutta and I. Maor, Phys. Rev. D 75, 063507 (2007).

[10] D. F. Mota, D. J. Shaw, and J. Silk, arXiv:0709.2227.

[11] L. R. Abramo, R.C. Batista, L. Liberato, and R.
Rosenfeld, J. Cosmol. Astropart. Phys. 11 (2007) 12.

[12] D. F. Mota and C. van de Bruck, Astron. Astrophys. 421, 71 (2004).

[13] N. J. Nunes and D. F. Mota, Mon. Not. R. Astron. Soc. 368, 751 (2006).

[14] W. Hu, Astrophys. J. 506, 485 (1998).

[15] J. Garriga and V. Mukhanov, Phys. Lett. B 458, 219 (1999).

[16] J. E. Gunn and J. R. Gott III, Astrophys. J. 176, 1 (1972).

[17] L. R. Abramo, R. C. Batista, L. Liberato, and R. Rosenfeld (work in progress).

[18] M. Manera and D. F. Mota, Mon. Not. R. Astron. Soc. 371, 1373 (2006).

[19] N. J. Nunes, A.C. da Silva, and N. Aghanim, Astron. Astrophys. 450, 899 (2006).

[20] R. R. R. Reis, Phys. Rev. D 67, 087301 (2003); 68, 089901 (2003).

[21] L. Amendola, I. Waga, and F. Finelli, J. Cosmol. Astropart. Phys. 11 (2005) 009.

[22] L. Amendola, F. Fineli, C. Burigana, and D. Carturan, J. Cosmol. Astropart. Phys. 07 (2003) 005. 